This is the post print version of an article that has been published in IEEE Journal of Biomedical and Health Informatics 2017, vol. 21, no.5, pp.1360-1366. The fnal version of record is available at http:// dx.doi.org/10.1109/JBHI.2016.2551862. Copyright (c) 2017 IEEE. Personal use is permitted. For any other purposes, permission must be obtained from the IEEE.

This document has been downloaded from TamPub.uta.fi The Institutional Repository of University of Tampere

\title{
On Biometrics with Eye Movements
}

\author{
Youming Zhang and Martti Juhola, Member, IEEE
}

\begin{abstract}
Eye movements are a relatively novel data source for biometric identification. When video cameras applied to eye tracking become smaller and more efficient, this data source could offer interesting opportunities for the development of eye movement biometrics. In the present article, we study primarily biometric identification as seen as a classification task of multiple classes, and secondarily biometric verification considered as binary classification. Our research is based on the saccadic eye movement signal measurements from 109 young subjects. In order to test the data measured, we use a procedure of biometric identification according to the one-versus-one (subject) principle. In a development from our previous research, which also involved biometric verification based on saccadic eye movements, we now apply another eye movement tracker device with a higher sampling frequency of $250 \mathrm{~Hz}$. The results obtained are good, with correct identification rates at $80-90 \%$ at their best.
\end{abstract}

Index Terms: Biometrics, eye movements, identification of persons

\section{INTRODUCTION}

Biometric identification has been researched extensively in pattern recognition and related fields during the last few decades. Technologies on based of fingerprints [1] and face images [1-4] have also been successfully used in commercial applications. These methods are still being developed, achieving even better results for biometric purposes. In addition, several other form of biometric data are being studied, such as iris [3,5-7], retina [8], palm, and ear images [9,10], which are seen as fairly stable data sources, even though aging may change the subject in the long run. Pupil sizes are used for human identification and verification [11]. Other sorts of biometric data sources are sometimes called behavioral features. Typically, these are physiological signals measured from subjects, such as EEG, ECG, and voice signals [12]. Furthermore, gait $[12,13]$ is an interesting alternative that is recognized from video streams of walking subjects. The use of EEG, ECG, and voice signals as biometric techniques may be quite difficult because they are not easy to interpret and may vary depending on the circumstances. For example, ECG measured at rest differs from that recorded immediately after rapid movement. All different modalities contain advantages and disadvantages with respect to their feasibility. For instance, it may depend on illumination and a subject's pose whether face images are applicable, and the dryness or cleanliness of the fingertips may determine whether good fingerprint images can be obtained.

Y. Zhang and M. Juhola are researchers at the School of Information Sciences, 33014 University of Tampere, Finland. (email: Martti.Juhola@sis.uta.fi)
For every modality, various shortcomings are recognized [13]. Thus, novel modalities have been introduced in recent years. For biometric verification and identification, novel biometric features are studied in an attempt to exploit information that originates from the brain and contains behavioral factors. A few years ago, we began to study eye movements for biometric verification or authentication, and we have obtained promising results [14-19]. We began our biometric research by seeing verification as a simpler problem than identification, because verification is a two-class classification task, whereas identification is a $c$-class classification task with $c$ possibly being a great number of subjects. In the current study, we extend our research to identification on the basis of saccadic eye movements by creating a new testing algorithm and using another eye movement measuring device with a higher sampling frequency than in our earlier research [14-18].

Eye movements have been proposed for biometrics using the optic stimulation from a moving spot [20]. Signal analysis has been applied by computing Cepstrum features in order to identify the subject. Eye movements were measured from 9 subjects with an eye tracking device on the basis of two small video cameras catching the movements of the pupil of each eye. Both a moving spot and images as stimulations have been used for 12 subjects [21]. Nevertheless, eye movements were found to be less useful for biometric identification than other factors, particularly the distance between the eyes of the subject. Eye movements from 41 subjects have been investigated by utilizing an optimization approach as an oculomotor plant model [22]. An eye movement technique has also been introduced for biometric identification by using face images as stimulations and graph matching methods for the eye movements recognized [23]. Measurements collected from 15 subjects have covered both horizontal and vertical directions using face images as stimulations. Minimal spanning trees for graphs were constructed, which were used for biometric identification and also applied statistical methods. Moreover, there are somewhat similar features [24] computed from saccadic eye movements to those in our prior research [14-19] and also employed in the present research. Twenty-two, 32, and 173 subjects were measured using three different set-ups [24].

Recently, a few novel articles have been published on eye movement biometrics. A method has been built on the basis of clustering to identify subjects with scan-path signals of eye movements [25] using a dataset of 32 subjects. Saccades and 
eye fixations have also been used together with iris images for the biometric identification of 22 subjects [26]. Saccadic eye movements and gaze fixations between them were applied from 34 subjects for user identification [27]. Eye movement signal were transformed into a decomposition form with a probabilistic representation of spatial and temporal features related to eye fixations on the basis of a large dataset of 200 subjects [28].

A biometric approach was developed for human identification and verification with eye blinks [29]. The electrooculographic (EOG) data of 25 healthy subjects were classified using different classifiers, such as Learning Vector Quantization, Discriminant Analysis, and Support Vector Machines.

Saccades are interesting as a biometric data source, because they are the fastest movements a human is able to perform. Their durations are approximately 0.1 to $0.4 \mathrm{~s}$ and the latency after a stimulation has been observed to be $0.2 \mathrm{~s}$ on average. Thus, a human is easily able to make one or two saccades per second. Most eye movements made are saccades, for example, while looking at immobile targets in an environment or while reading a text. The brain, particularly the brainstem, controls all processing. Once a saccade is underway, a subject cannot alter it at will [30]. Therefore, a large amplitude saccade may not always hit a target very accurately. Consequently, the exact replication of saccades is virtually impossible. This property is very useful for biometric verification and identification with regard to counterfeits and the recognition of a subject in situ. Most saccades are of less than perhaps $10^{\circ}$ in normal life when a subject looks at surrounding objects or reads a text. Nevertheless, it is easy to make much a larger saccade - even up to $60-90^{\circ}$ - by moving the gaze, in other words, rotating one's eyeballs to focus on another target. Saccades of large amplitudes have been used in medicine since the 1960s, because they can reveal diseases better than saccades of small amplitudes [30-32]. Therefore, we assumed that large saccades may also reveal better differences between individuals.

\section{Signal Data}

To measure saccadic eye movements (i.e. their amplitudes) we used an eye movement camera system from EyeLink (SR Research, UK), which measures eye movements of $\pm 30^{\circ}$ by tracking the location of a subject's pupil from every successive image of a video stream and computing the difference between the successive locations. The system, which contains two cameras, is attached with the headband.

At the beginning, calibration of the camera system is accomplished for each subject before recording eye movements by moving a light dot on a computer screen. The task of the subject is to follow the light dot, but not to move his or her head. Horizontal moves of the stimulation dot are controlled by the computer by slightly varying the durations of the intervals between the jumps to make stimulations random for subjects, thus the subjects do not attempt to anticipate stimulation movements. The subject is seated in a fixed chair, maintaining a constant distance from the computer screen. The same stimulation sequence with varying stimulation amplitudes and intervals is used for every subject to make measurements comparable between individuals. The sampling frequency is $250 \mathrm{~Hz}$.

Saccadic eye movements are easy to stimulate for biometrics, and they can be produced in many ways. We applied a simple stimulation formed by a small light dot jumping horizontally on a computer screen in front of the sitting subject (Fig. 1). The distance of the eyes from the screen was fixed in order to generate the same stimulation sequence with the same stimulation amplitudes (angles) for every subject. Such stimulations have been utilized in neurological and other medical tests for decades [30-32]. We have exploited this straightforward method in order to collect a few dozen saccades of the same large amplitude for identification. Fig. 2 shows a signal example. A statistically large enough dataset is important when applying machine learning methods for biometric identification for any purpose. In principle, the more data per subject, the more information is obtained from the subject and this may improve the identification results. It is also necessary for comparing data between different subjects, as the data are the basis for identification and verification.

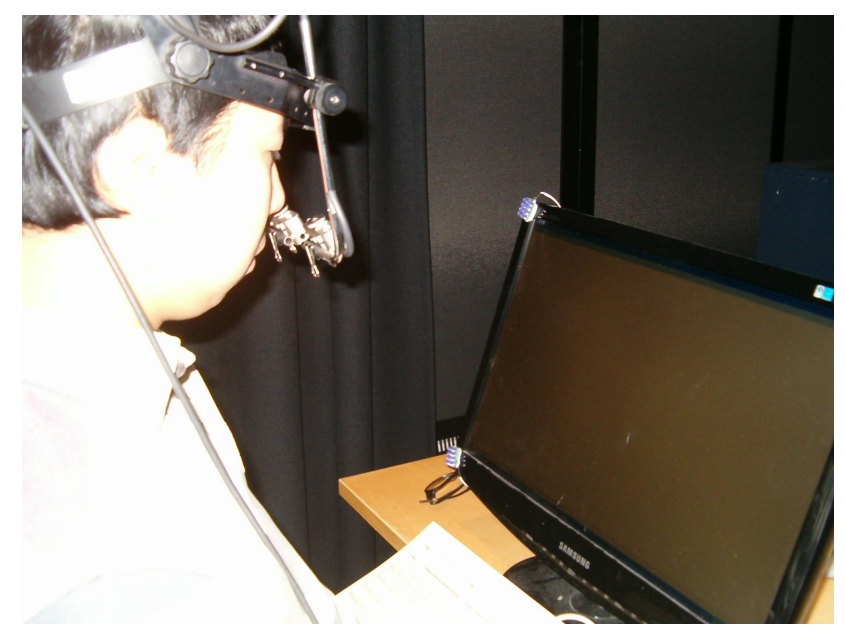

Fig. 1. The stimulation and measurement set-up where the subject follows a horizontally jumping light dot on the screen with his gaze for $60 \mathrm{~s}$ without moving his head or closing his eyes.

A sequence of stimulation angles takes $60 \mathrm{~s}$ and contains 51 stimulation angles, of which 30 were the large ones $\left(51^{\circ}\right)$ used for the tests. An example is shown in Fig. 2. Large amplitudes have been applied to biometric identification, because we may assume that they give larger differences of saccadic features between subjects than saccades of small amplitudes. The larger the amplitude, the larger values are attained for most saccadic features (described in Section IV). Therefore, differences in individuals' saccades may be easier observed. Furthermore, the approximate calibration resolution of the device used was $0.5^{\circ}$, meaning the larger amplitude, the better. This favors the use of large amplitudes, because the maximal error of $\pm 0.5^{\circ}$ is then smaller in percentage terms for the large amplitude of $51^{\circ}$. 


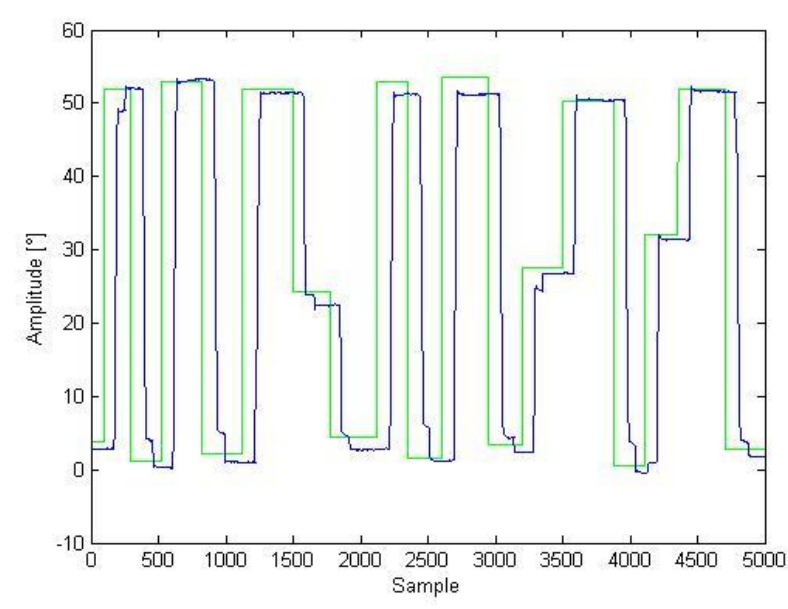

Fig. 2. A (blue) saccadic eye movement signal where the saccades followed the (noiseless) stimulation signal. The down direction on the vertical axis corresponds to an eye movement to the left, and the up direction to the right. The length of the signal segment is $20 \mathrm{~s}$.

For the large saccades of $51^{\circ}$, the direction is either from the left to the right edge or from the right to the left, with almost the entire width of the computer screen used. Other stimulation angles are smaller and also enable other alternatives (see Fig. 2) and their directions alter unsystematically. The intervals of roughly $1 \mathrm{~s}$ between stimulation movements vary slightly. The purpose of the amplitude and interval variations is to make stimulations difficult to anticipate, since anticipated or guessed saccades would not respond to the actual stimulations. The amplitude and interval variations are random from the viewpoint of the subject and thus eliminate the learning effect. Actual responses to real stimulations known to the researchers are desired to enable the computation of latency between stimulations and saccades, and the accuracy of saccadic amplitudes related to stimulations. In a way, anticipated eye movements would be more or less random, not responses to stimulation movements.

Four separate measurements were recorded in succession from every subject, because saccades of the same stimulation amplitude may vary a little in their features. Of course, we also needed more than one measurement to use them in different ways in training and test sets employed with classification methods to run biometric identification experiments. We measured the saccades of 81 male and 28 female volunteers. The subjects' average age was $25.2 \pm 6.0$ years. We measured 120 saccades of the largest amplitude from every subject.

\section{DATA ANALYSIS}

First, a signal was filtered through a standard median filter of the window length of three samples. As usual, the eye movement tracker contains two cameras, one for each eye. Thus, the less noisy signal is chosen from the two measured from the subject. Typically, the noise present is very slight. Nevertheless, a single eye movement signal suffices for biometric identification. Next, saccades as responses to the stimulation sequence were recognized according to the first derivative approximated from the chosen eye movement position signal. This corresponds to the angular velocity of the eye. In order to recognize the beginning of a saccade, we applied a threshold of $50 \%$ and $10 \%$ for the end. The latter is less because, normally, the beginning segment of a saccade is faster (steeper) than its end segment. Furthermore, all stimulation movements have been searched for directly from the virtually noiseless stimulation signal as sharp steps.

In addition to the velocity signal, the angular acceleration and deceleration signal were approximated as the second derivative, exemplified by Fig. 3. Both the velocity signal and the acceleration-deceleration signal were also exploited to compute some saccadic features (described in the following section).

\section{SACCAdic Features COMPUTED}

Eight features have been dealt with in order to characterize each subject, in other words, for biometric identification. The present features were chosen since they measure the subject's physiology and behavior in regard to eye movements while following the jumping stimulation light dot in the visual field. These features are applied in medical fields such as otoneurology and physiology [30,32].

Fig. 3 shows the schema for the features, of which three depend directly on time. Latency $l$ equals the time difference from the saccade beginning $b_{r}$ to the stimulation beginning $b_{s}$. Duration $d$ is the difference from the saccade end $e_{r}$ to its beginning $b_{r}$. Time to maximum velocity $t_{m v}$ is equal to the difference from the location of the maximum velocity $p^{\prime}(t)$, determined by the velocity signal, to that of saccade beginning. Two features relate to the saccade angle. Amplitude $a_{r}$ is the (absolute) angular difference between the locations of the saccade $p\left(e_{r}\right)$ end and beginning $p\left(b_{r}\right)$. Accuracy $c$ is the difference between amplitudes of a stimulation amplitude $a_{s}$ and its responsive saccadic amplitude $a_{r}$. This is positive when the subject's brain produces a slightly smaller angle than its large stimulation, in the other words, the gaze does not hit the target precisely. It is seldom negative - i.e. the saccade is rarely too large. Ultimately, maximum angular velocity $p^{\prime}(t)_{\max }$, maximum angular acceleration $p$ '” $(t)_{\max }$, and maximum deceleration $\mid p$ '” $(t)_{\text {min }} \mid$ (the absolute value of the minimum) were computed.

\section{IDENTIFICATION AND VERIFICATION EXPERIMENTS}

There are four signals from every subject. Every signal consists of 30 large amplitude saccades applied to our experiments. One signal was taken for testing and the other three, with a total of 90 saccades, were taken for training. 


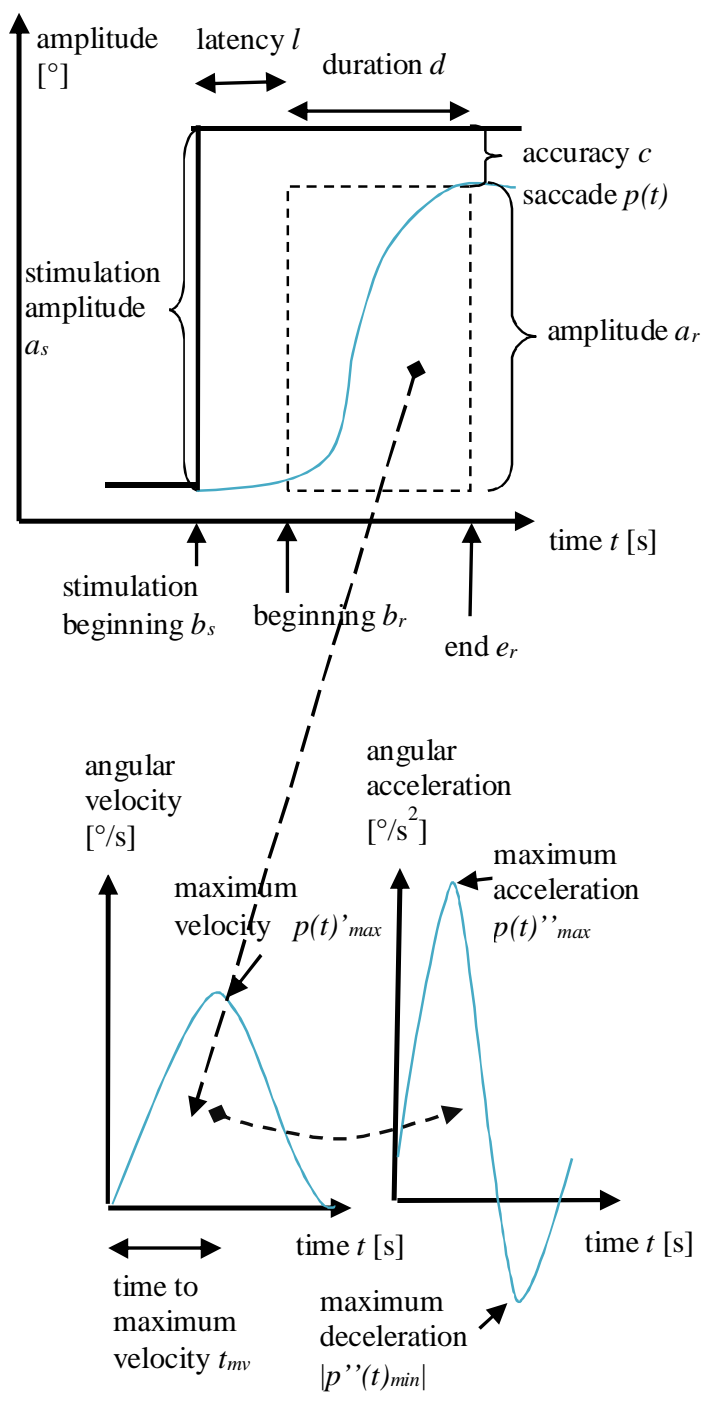

Fig. 3. A hypothetical saccade (above) and its velocity and accelerationdeceleration curves (below) along with saccade features to be computed: Latency, duration, time to maximum velocity, amplitude, accuracy, maximum velocity, acceleration, and deceleration.

As frequently used for classification with machine-learning methods, we followed the one-versus-one principle as follows: There are $n(n-1) / 2$ pairs for $n=109$ subjects. A pair of two subjects includes two times 90 saccades equal to 180 saccades from two subjects for training. For all these models, an authenticated subject (one by one among all subjects) was tested with his or her 30 test saccades, but his or her other 90 saccades were used for building a model for classification. These $n-1=108$ runs have been made for all 109 subjects. In Algorithm 1, a winner subject is the one who has received the majority of scores and could get no more than the maximum of 108 scores. Ties were also possible, i.e., more than one subject may have obtained the same highest score smaller than or equal to $108 / 2$. The algorithm checks whether the authenticated subject obtains the most scores.
Types of classifiers and their parameter set-ups have been chosen according to the test results obtained in our prior biometric verification studies [14-19], in which their tests have been executed extensively with several parameter selections and several other classification algorithms not used here.

\section{Algorithm 1 for identification}

\author{
All subjects $n=109$; \\ For $i=1: n$ subjects, the $i$ th being the authenticated one at a time \\ $k=m=0$;
}

Select one measurement (30 saccades) from the authenticated subject to be test saccades.

For $j=1: n(n-1) / 2$ pairs of subjects,

Build a classification model on the basis of a subject pair: three measurements from each, i.e., $2 \times 90$ saccades, in the training set.

Compute a test by classifying with 30 test saccades.

The winner subject obtains a score of 1 and the loser a score of 0 . A tie being possible, in this case the winner subjects obtain 1 /number of the winners ( 2 in practice).

\section{End}

If the authenticated subject received the highest scores,

$$
k=k+1 \text {; }
$$

If the authenticated subject was the only one to receive the highest scores (no tie),

$m=m+1$

End

$v=(k / n) \cdot 100 \%$

$u=(\mathrm{m} / \mathrm{n}) \cdot 100 \%$

Computing the tests in the above way, quantity $u$ gives the rate of surely correct identifications, because there is no tie. The larger value of $v$ manifests possibly correct identifications because of ties. An occurrence of ties, i.e. an equal number of the highest scores for more than one subject, means that we are not sure whether the correct subject would be selected from among those inferred to be equally plausible.

We programmed all computing with Matlab. Running times of some classifier types for 109 subjects took a long time: 2 hours for logistic discriminant analysis (LDA), 4 hours for each of the support vector machine (SVM) alternatives, 10 days for multilayer perceptron (MLP) networks, and 21 days for radial basis function (RBF) networks with a $3 \mathrm{GHz}$ machine. Therefore, we have not repeated experiments by alternating the test sequence through all four measurements for 109 subjects, but have only run it using one test sequence for each subject. The time complexity of the algorithm is $O\left(n^{3}\right)$, but in an actual application it would be less, $O\left(n^{2}\right)$, since only one subject is 
then due to be identified at a time.

As a result of the long running times, we also tested biometric identification by experimenting with a smaller set of $n$ subjects equal to 20 and repeated this for 10 iterations by randomly choosing 20 of the subjects at a time from all 109. Finally, we calculated the averages of 10 iterations.

In order to do the verification, identification is first run to detect a winner, i.e. probably the correct subject. Nevertheless, in the worst case, a tie is possible, producing more than one winner candidate: this has to be taken into account in the algorithm. Because the two test series run above have not dealt with possible impostors, we have continued by experimenting with the verification mode after the preceding experiments, as defined above, with 20 randomly taken subjects repeated for 10 iterations. For verification (Algorithm 2), which is a two-class classification task, every training set has been built between saccades of the authenticated subject and those of other subjects by using the equal number of saccades from these two classes. Intruders, labelled "impostors", are simulated by taking them outside the preceding group of 20 subjects.

\section{Algorithm 2 for verification}

$U=\{$ all 109 subjects $\}$;

$\mathrm{c}_{1}=\mathrm{c}_{2}=0$

For $i=1: 10$ iterations,

Select randomly $A=\{20$ to be subjects for model building and tests $\}$ and $B=\{20$ to be impostors $\}$ from $U$.

For each $t$ in $A+B$,

Take one measurement (30 saccades) of $t$ to be test set $T$.

Run Algorithm 1 with test set $T$ and training set from $A$.

If more than one winner candidate (tie) is obtained, this is a "no match" and skip the following verification.

Verification:

Make training set $E$ : (1) If $t$ is from $A$, take the other 3 measurements ( 90 saccades) of $t$. Otherwise if $t$ is from B, take 3 measurements of some case from $A$. (2) Take 90 saccades by selecting randomly 10 saccades from a measurement (30 saccades) of each of the 9 subjects from those of $U-A+B$ (69 subjects).

Train a classifier with $E$ for two classes: the authenticated subject and those other from $U-A+B$.

Run verification with $T$.

If the class of the authenticated subject gets enough support ( $\geq 25$ scores out of 30 corresponding to the test saccades), a winner candidate is found. Otherwise this is "no match".

For no match and authenticated $t$, we get a false negative (false rejection).

For no match and impostor $t$ we get a true negative: $c_{2}=c_{2}+1$.
For impostor $t$ and the winner candidate found, we get a false positive (false acceptance).

For authenticated $t$ and the winner candidate found, and these are the same subject, we get a true positive: $c_{1}=c_{1}+1$.

For authenticated $t$ and the winner candidate found, but these are different, we also get a false positive.

End

Compute average true positive $c_{1} /(10 * 20)$ and true negative $c_{2} /(10 * 20)$.

\section{RESULTS OF EXPERIMENTS}

In order to classify saccades, we have applied logistic discriminant analysis, linear, the quadratic and polynomial of the $3^{\text {rd }}$ degree support vector machines, support machine with a radial basis kernel function (RBF) with parameter $\sigma$ equal to 4 , radial basis function networks with normalized data $(\mathrm{Sp}=15$ and goal $=0.12$ ), and multilayer perceptron networks trained with 8 nodes of one hidden layer according to the LevenbergMarquardt algorithm. The parameter values mentioned are selected as the best with regard to the results from among several alternatives along with the experiments from our previous research [14-19].

TABLE I

IDENTIFICATION RATES (\%) GIVEN BY SVM CLASSIFIERS IN WHICH $u$ IS THE SURELY CORRECT RESULT AND $v$ IS POSSIBLY CORRECT BECAUSE OF TIES.

\begin{tabular}{|c|c|c|c|c|}
\hline \multirow[t]{3}{*}{ Classifier } & Linear & Q Quadratic & $\begin{array}{l}\text { Polynomial } \\
\end{array}$ & $\overline{\text { SVM with RBF }}$ \\
\hline & SVM & SVM & SVM & kernel \\
\hline & $u$ and $v$ & $u$ and $v$ & $u$ and $v$ & $u$ and $v$ \\
\hline $\begin{array}{l}\text { Identification: } \\
109 \text { subjects }\end{array}$ & 63.367 .0 & $79.8 \quad 84.4$ & 80.785 .3 & $83.5 \quad 86.2$ \\
\hline $\begin{array}{l}\text { Identification: } \\
20 \text { subjects for } \\
10 \text { times }\end{array}$ & $\begin{array}{lll}83.0 & 87.0\end{array}$ & $90.0 \quad 93.5$ & 86.090 .5 & 91.093 .5 \\
\hline
\end{tabular}

TABLE II

IDENTIFICATION RATES (\%) GIVEN BY LOGISTIC DISCRIMINANT ANALYSIS (LDA), RADIAL BASIS FUNCTION (RBF) AND MULTILAYER PERCEPTRON (MLP) NETWORKS IN WHICH $u$ IS THE SURELY CORRECT RESULTS AND $v$ POSSIBLY CORRECT BECAUSE OF TIES.

\begin{tabular}{|c|c|c|c|}
\hline Classifier & LDA & RBF & MLP \\
\hline & $u$ and $v$ & $u$ and $v$ & $u$ and $v$ \\
\hline $\begin{array}{l}\text { Identification: } \\
109 \text { subjects }\end{array}$ & 67.971 .6 & $\begin{array}{ll}83.5 & 86.2\end{array}$ & 75.285 .3 \\
\hline $\begin{array}{c}\text { Identification: } 20 \\
\text { subjects for } 10 \\
\text { times }\end{array}$ & $85.0 \quad 90.0$ & 91.093 .5 & $82.5 \quad 90.0$ \\
\hline
\end{tabular}


TABLE III

AVERAGE VERIFICATION RESULTS (\%) OF ALGORITHM 2 FOR THE 10 SETS OF 20 SUBJECTS WITH FALSE REJECTION RATES (FRR) AND FALSE ACCEPTANCE RATES (FAR).

\begin{tabular}{ccc}
\hline \hline Classifier & FRR & FAR \\
\hline Linear SVM & 46.5 & 38.5 \\
Quadratic SVM & 21.5 & 28.5 \\
Polynomial SVM & 36.5 & 11.5 \\
SVM with RBF kernel & 24.5 & 34 \\
LDA & 46.5 & 30.0 \\
RBF & 24.5 & 34.0 \\
MLP & 41.2 & 26.9 \\
\hline \hline
\end{tabular}

Identification rates for the 109 subjects are shown in percentages in Tables I and II. They have been computed with Algorithm 1 according to the one-versus-one (subject) principle. Comparing and looking for the best results, we find that the results of SVM with the RBF kernel in Table I are equally good as those of the radial basis function network in Table II. The results of the quadratic SVM are the next best, followed, in the descending order, by the polynomial $\left(3^{\text {rd }}\right.$ degree) SVM, multilayer perceptron, logistic discriminant analysis, and linear discriminant analysis. The difference between the best and worst is over $10 \%$.

Table III consists of the results of the other experimental set-up: first identification and second average false rejection rates and false acceptance rates for verification after identification. This was performed with 20 randomly selected subjects for 10 iterations by finally computing averages for all classification methods employed. Looking at the mean of FRR and FAR, the polynomial SVM is the best and the quadratic SVM is almost equally as good; SVM with the RBF kernel and the radial basis function network come equal third, followed by the multilayer perceptron network, logistic discriminant analysis, and linear SVM.

To compare the results of identification and verification, the orders of the best classification methods vary, while multilayer perceptron network, logistic discriminant analysis, and linear SVM are the same in both.

\section{DISCUSSION}

According to the results, we may conclude that it is efficient to apply methods other than a multilayer perceptron network, logistic discriminant analysis, and linear SVM. In other words, it seems to best to choose from quadratic SVM, $3^{\text {rd }}$ degree SVM, SVM with an RBF kernel, or a radial basis function network.

Other researchers in the field of eye movement biometrics have reported results as follows: average false acceptance rate (FAR) from $1.4 \%$ to $17.5 \%$ and average false rejection rate (FRR) from $12.6 \%$ to $28.9 \%$ [20] for 9 subjects, and an identification rate of $83 \%$ for 12 subjects [21]; FAR $5.4 \%$ and FRR $56.6 \%$ for 41 subjects [22]; an equal error rate (EER) of nearly $30 \%$ for 15 subjects [23]; EER around 35\% for 173 subjects [24]; the best EER of $10.8 \%$ [28] for 200 subjects; accuracy of $63 \%$ for 32 subjects [26], the best accuracy of $43.1 \%$ for 22 subjects [26]; and an accuracy of $33.3 \%$ for 34 subjects [27]. The results obtained in the present research are at least equally good compared with these preceding studies. Nevertheless, the computational approaches of identification and verification, eye movement camera systems, methods of stimulating eye movements, and numbers of subjects employed have varied greatly. Therefore, no rigorous conclusions can be drawn between the earlier studies and our current results. Still, one can criticize the very small numbers of subjects in several earlier studies. Those with less than 30-40 subjects can be seen as preliminary investigations purely on statistical grounds.

A researcher with a thorough knowledge of machine learning algorithms could show the weaknesses of how inadequately scarce tests have obviously been implemented in some of the previous research [20-27], and some descriptions of the test procedures have been very narrow. In order to achieve statistically reliable and credible results, the validation task has to be done thoroughly and it must follow the correct principles of testing in machine learning. Sometimes a straightforward split called hold-out validation $[33,34]$ has obviously been applied, perhaps without remembering that it is only reasonable and usable for relatively great numbers of subjects, such as a few thousand. Of course, this depends on the properties of the data, e.g., how many variables are used. In addition, several models have to be built because normally some of them do not give reliable results; it is only after computing many models that their average results justify credible overall results and fitting conclusions. Sophisticated machine-learning algorithms such as multilayer neural networks probably cannot learn from small numbers of subjects and build statistically credible models because they have to train numerous weight values or other parameter values for modeling. In such cases, the simplest techniques, such as $k$-nearest neighbor searching, have to be utilized.

Altogether, we may assume that the results attained can still be improved in future research and that saccades could be used jointly with other biometric data sources even for biometric identification. A probable advantage in the future will be the development of smaller - but more efficient and accurate - eye movement cameras.

Although the identification task pursued on the basis of saccadic eye movements is more difficult than our previous verification experiments with saccades [14-19] because of the character of the $c$-class $(c>>2)$ classification of identification compared to the two-class classification of verification, we have obtained better identification rates than expected in this first identification attempt. However, the occurrence of impostors is still a difficult issue. Clearly, more extensive data sets than used here might be complicated for identification 
based on saccades. For real-life applications in the future, we expect that eye movement biometrics will be applied jointly with some other biometrics, such as face or pupil images or fingerprints.

\section{ACKNOWLEDGMENTS}

The authors wish to thank Prof. K.-J. Räihä, P. Majaranta, Ph.D., and O. Špakov, Ph.D., of the School of Information Sciences, University of Tampere for their aid in the acquisition of the eye movement data.

\section{REFERENCES}

[1] A.K. Jain, J. Feng, and K. Nandakumar, "Fingerprint matching," Computer, vol. 43, no. 2, pp. 36-45, 2010.

[2] A. Pentland and T. Choudbury, "Face recognition for smart environments," Computer vol. 33, no. 2, pp. 50-55, 2000.

[3] A. Ross and A. Jain, "Information fusion in biometrics," Pattern Recogn. Letters, vol. 24, no. 13, pp. 2115-2125, 2003.

[4] R. Chellappa, P. Sinha, and P.J. Phillips, "Face recognition by computers and humans," Computer, vol. 43, no. 2, pp. 46-55, 2010.

[5] M. Negin, T.A. Chmielewski, M. Salganicoff, T.A. Camus, U.M. Cahn von Seelen, P.L. Venetianer, and G.G. Zhang, "An iris biometric system for public and personal use," Computer, vol. 33, no. 2, pp. 70-75, 2000.

[6] A. Ross, "Iris recognition: the path forward," Computer, vol. 43, no. 2, pp. 30-35, 2010

[7] N.D. Kalka, J. Zuo, N.A. Schmid, and B. Cukic, "Estimating and fusing quality factors for iris biometric images," IEEE Trans. Syst., Man, Cybern. A, Syst., Humans, vol. 40, no. 1, pp. 509-524, May 2010.

[8] W. Barkhoda, F. Akhlaqian, M.D. Amiri, and M.S. Nouroozzadeh, "Retina identification based on the pattern of blood vessels using fuzzy logic," EURASIP J. Adv. Signal Process., vol. 113, 9 p., 2014.

[9] K. Faez, S. Motamed, and M. Yaqubi, "Personal verification using ear and palm-print biometrics," Proc. IEEE Intern. Conf. Systems, Man and Cybernetics, 2008, pp. 3727-3731.

[10] J.D. Bustard and M.S. Nixon, "Toward unconstrained ear recognition from two-dimensional images," IEEE Trans. Syst., Man, Cybern. A, Syst. Humans, vol. 40, no. 1, pp. 486-494, May 2010.

[11] N. Nugrahaningsih and M. Porta, "Pupil size as a biometric trait," LNCS 8897, Springer, Heidelberg, pp. 222-233, 2014

[12] G. Goudelis, A. Tefas, and I. Pitas, "Emerging biometric modalities: a survey," J. Multimodal User Interfaces, vol. 2, pp. 217-235, 2008

[13] T. Connie, M.K.O. Goh, and A.B.J. Tech, "A Grassmann graph embedding framework for gait analysis," EURASIP J. Adv. Signal Process., vol. 15, 17 p., 2014.

[14] Y. Zhang, J. Rasku, and M. Juhola, "Biometric verification of subjects using saccade eye movements," Int. J. Biometrics, vol. 4, no. 4, pp. 317 337, 2012.

[15] M. Juhola, Y. Zhang, and J. Rasku, "Biometric verification of a subject through eye movements," Comp. Biol. Med., vol. 43, no. 1, pp. 42-50, 2013.

[16] Y. Zhang and M. Juhola, "Biometric verification of a user based on eye movement," Int. J. Biometrics, vol. 6, no. 2, pp. 106-124, 2014.

[17] Y. Zhang, J. Laurikkala, and M. Juhola, "Biometric verification of a subject with eye movements, with special reference to temporal variability in saccades between a subject's measurements," Int. $J$. Biometrics, vol. 6, no. 1, pp. 75-94, 2014.

[18] Y. Zhang and M. Juhola, "On biometric verification of subjects using saccade eye movement data mining," Proc. Second Int. Conf. Adv. Inf. Mining Management, pp. 85-90, 2012.

[19] Y. Zhang and M. Juhola, "On applying signals of saccade eye movement for biometric verification of a subject," Proc. $8^{\text {th }}$ International Conference on Mass Data Analysis of Images and Signals, pp. 78-92, 2013.

[20] P. Kasprowski and J. Ober, "Eye movements in biometrics," Biometric Authentication: Int. Workshop, Prague, Czech Republic, 2004, LNCS 3087, pp. 248-258.
[21] R. Bednarik, T. Kinnunen, A. Mihaila, and P. Fränti, "Eye-movements as a biometric," Image Analysis, LNCS 3540, Springer, Heidelberg, pp. 780789,2005

[22] O.V. Komogortsev, S. Jayarathna, C.R. Aragon, and M. Mahmoud, "Biometric identification via an oculomotor plant mathematical model," Proc. 2010 Symp. On Eye-Tracking Research and Applications, ACM, New York, NY, USA, pp. 57-60, 2010.

[23] I. Rigas, G. Economou, and S. Fotopoulos, "Biometric identification based on the eye movements and graph matching techniques," Pattern Recogn. Letters, vol. 33, pp. 786-792, 2012.

[24] C.D. Holland and O.V. Komogortsev, "Complex eye movement pattern biometrics: the effects of environment and stimulus," IEEE Trans. Inf. Forensics Security, vol. 8, no. 12, pp. 2115-2126, 2013.

[25] B. Tripathi, V. Srivastava, and V. Pathak, "Human recognition based on oculo-motion characteristics," Proc. AFRICON 2013, IEEE, pp. 1-5, 2013.

[26] A. Darwish and M. Pasquier, "Biometric identification using the dynamic features of the eyes," Proc. IEEE Sixth Int. Conf. on Biometrics: Theory, Applications and Systems, p. 1-6, 2013.

[27] M.R.M. Motlagh and P. Bours, "User identification based on eye gaze data," Norsk Informatikkonfereanse, NIK-2014, pp. 1-9, 2014. http://www.nik.no/

[28] I. Rigas and O. Komogortsev, "Biometric recognition via probabilistic spatial projection of eye movement signals," IEEE Trans. Inf. Forensic Security, vol. 9, no 20, pp. 1743-1754, 2014.

[29] M. Abo-Zahhad, S.M. Ahmed, and S.N. Abbas, "A novel biometric approach for human identification and verification using eye blinking signal," IEEE Signal. Process. Letters, vol. 22, no. 7, pp. 876-880, 2015.

[30] R.J. Leigh and D.S. Zee, The Neurology of Eye Movements, fourth ed. U.K. Oxford University Press, London, 2006.

[31] A.T. Bahill, A. Brockenbrough, and B.T. Troost, "Variability and development of a normative data base for saccadic eye movements," Invest. Ophthalmol. Vis. Sci., vol. 21, no. 1, pp. 116-125, 1981.

[32] M. Juhola, V. Jäntti, and I. Pyykkö, "Effect of sampling frequencies on the maximum velocity of saccadic eye movements," Biol. Cyber., vol. 53, no. 2, pp. 67-72, 1985.

[33] K.J. Cios, W. Pedrycz, R.W. Swiniarski, and L.A. Kurgan, "Data Mining, A Knowledge Discovery Approach,” Springer Science+Business Media, LLC, New York, USA, 2007

[34] C.M. Bishop, "Pattern Recognition and Machine Learning," Springer Science+Business Media, LLC, New Delhi, India, 2013. 